\title{
A Clinical Study of Cervical Lymphadenopathy
}

\author{
Dr. K. Srinivasa Rao ${ }^{1}$, Dr. K. Venkata Vigneswara Rao ${ }^{2}$ Dr. K.S.Raja Rao ${ }^{3}$ \\ ${ }^{1}$ (M.S[Gen Surgery] Asst Professor In Department Of General Surgery GGH/GMC- \\ GUNTUR.,A.P..,INDIA) \\ ${ }_{2}^{2}$ (M.S[Gen Surgery] Asst Professor In Department Of General Surgery GGH/GMC- \\ GUNTUR.,A.P..,INDIA \\ ${ }^{3}$ Professor In Department Of General Surgery, GGH/GMC, GUNTUR.A.P
}

\begin{abstract}
The aim of study is to know the etiology of cervical lymphadenopathy and to study clinical presentation and clinical course and management of cervical lymphadenopathy.

Patients And Methods The study was conducted in government general hospital /Guntur Medica! College, Guntur between the period of 3 years i.e. from November 2012 to October2014. The present study includes 72 patients in both outpatients and in patients in the Department of General Surgery. Results: Of the 72 patients we studied, $66.66 \%$ are found to be having Cervical Lymphadentis due to Tuberculosis and $18.5 \%$ are due to Reactive Cervical Lymphadenitis 5,33\% are due to secondaries, $4.16 \%$ are due to Hodgkin's Lymphoma and $1.38 \%$ are due to Non-Hodgkin's Lymphoma.

Conclusion: Of the 72 cases studied, tuberculosis had the maximum incidence [66.66\%], followed by reactive type of lymph nodes[18.5\%]. They were put on anti tubercular drugs for 6 months, recovery was 100\%.The cheapest and the most reliable method of diagnosis was fine needle aspiration cytology.
\end{abstract}

Keywords: Cervical Lymphadenopathy (CLN), Tuberculosis (TB), Fine Needle Aspiration Cytology(FNAC),Radiotherapy (RT), Anti Tuberculosis Treatment (ATT).

\section{Introduction}

Cervical Lymphadenopathy is a common presentation in the course of a number of diseases. This condition is generally not a disease by itself; rather, it may be a symptom of many possible underlying problems. The diseases can be neoplastic or inflammatory, and include lymphoma, metastatic carcinoma, infectious diseases, tuberculosis, etc. Since cervical lymphadenopathy is not a manifestation of a single disease, diagnosis becomes very difficult and moreover, it requires a strong knowledge about the differential diagnosis of cervical lymphadenopathy. Most patients can be diagnosed on the basis of a careful history and physical examination. This study is presents a methodical clinical approach and the treatment to patients presenting with cervical lymphadenopathy.

Lymph nodes which are interposed throughout the course of collecting lymphatic channels, vary from a millimeter to a centimeter or more in size. They act as filtering mechanism within the host that restricts propagating malignant cells and resists spread of infection.

For, a surgeon lymph node is an index of spread of infection or malignancy. Cases of lymphadenopathy are common in our country. There are many causes of enlargement of lymph nodes. Lymph node biopsies are' the only certain means of establishing the diagnosis. The microscopic interpretation of abnormal lymph node is extremely difficult. More errors are made on the lymph node than any other organ in the body. Of the many causes for cervical lymphadenitis, tuberculosis is still a common cause for lymphadenitis. In generalized lymphadenopathy, the most likely diagnoses are probably tuberculosis and Hodgkin's disease.

\section{Patients And Methods}

Patients

In this present clinical study of cervical lymphadenopathy 72 cases have been evaluated. The patients were seen as Out-patients \& In-patients in six units of Dept. of General Surgery at Government General Hospital, Guntur, over a period of 2 years from November 2012 to OCTOBER 2014.

\section{Methods}

The clinical findings of all cases which presented during this period were entered and investigations were tailored to each patient clinical finding to confirm the diagnosis. The required baseline investigations viz. FNAC, Excision biopsy \& E.N.T. examination were carried out and treatment advised accordingly. Since 
cervical lymphadenopathy is a common disease which presents to the Surgery dept. a prospective study was conducted in the above mentioned period. All the patients were asked to attend the Surgical OP for follow up after discharge and necessary advice given.

Inclusion Criteria

a) Patients more than 12 years age

b) OPD cases

Exclusion Criteria

a) Very sick and ill

b) Having abscess and discharges

c) On chemotherapy / radiotherapy

\section{Observations And Results}

The present study includes 72 patients, both outpatients as well as inpatients at the surgical OP/wards Guntur Medical College \& Govt General Hospital between November 2012 and October 2014. In the present series 72 cases were evaluated for cervical lymph node enlargement. All cases were taken for Fine Needle Aspiration Cytology (FNAC), but in 6 cases FNAC was inconclusive. Excisional biopsy was performed in all 72 cases and these cases were analyzed in detail. Maximum number of cases 59 (81.93\%) were between 12-40 years age group and the male to female ratio was $1.32: 1$. Out of these 72 cases, 48 cases $(66.66 \%)$ were confirmed as tubercular, 13 cases $(18.05 \%)$ as reactive lymphadenitis, 3 cases $(4.16 \%)$ as chronic non-specific lymphadenitis, 4 cases as secondaries and 4 cases as lymphoma. Thus among the lesions affecting neck lymph nodes tubercular etiology was the most common. The causes of cervical lymphadenopathy were broadly classified as neoplastic and non-neoplastic lesions.

\section{Treatment}

In the present study all the 48 cases proved as tubercular were treated with DOTS as per the RNTCP guidelines from the District TB centre at our hospital.

As per the WHO guidelines, tubercular lymphadenitis comes under category-II for which the treatment regimen is as follows:

Intensive phase - 2 months Isoniazid, Rifampicin and Pyrazinamide - 2(HRZ) 3

Continuation phase - 4 month Isoniazid and Rifampicin - 4(HR)3

Of the 48 tubercular cases, 3 cases had active pulmonary lesion (new sputum positive) and as per WHO guidelines comes under category-I. These patients were treated with 2(HRZE)3 4(HR)3 regimen.

The patients who presented with abscess were drained surgically and those presented with sinus underwent excision of sinus in addition to chemotherapy.

Out of the 72 patients 4 cases did not come for regular follow-up. Of the 68 cases who came for follow-up regularly, 55 cases the lymphnodes resolved uneventfully. In the remaining 13 cases, 4 cases developed fresh lymphnodes which later resolved on continuing the treatment. 2 cases developed abscess which was evacuated. At the end of therapy, 4 cases $(5.5 \%)$ had residual lymph nodes. The initial treatment for the 16 cases diagnosed as reactive and chronic non- specific lymphadenitis commenced with antibiotics which are active against gram positive bacteria In the present study, a 10-15 days course of penicillin group or macrolide group of antibiotics was prescribed and the clinical course observed. Those presenting with suppurative lymphadenitis underwent incision \& drainage (4 cases). Appropriate cultures were obtained. In 1 case no response was seen and the lymphnode was excised under local anesthesia.

Of the 4 cases diagnosed as malignant secondaries in cervical lymph nodes, 1 case had primary in the larynx, 1 each from Sq cell carcinoma Lower esophagus, Carcinoma tongue and Carcinoma Pyriform fossa . One case with primary in the larynx had a massive (>6) bilateral nodes (fixed), was inoperable. Remaining cases were referred to Radiotherapy, 4 cases of lymphomas were referred to Department of Radiotherapy, Government General Hospital, Guntur.

\section{Discussion}

The discussion is mainly on analysis and observations made regarding presenting symptoms, clinical behavior, signs, investigations, management and post-operative events in 72 cases of cervical lymph node enlargement attending to Govt general hospital and Guntur Medical College, Guntur during a period of two years. In the present study, out of 72 cases of cervical lymphadenopathy, 64 were non neoplastic lesions (88.88\%) and 08 $(11.11 \%)$ were neoplastic lesions.. In the present series, tuberculosis accounts for $66.66 \%$ of cases, $4.16 \%$ turned out to be chronic non-specific lymphadenitis and $18.05 \%$ reactive lymphadenitis. Among the neoplastic lesions, malignant secondaries accounted for $5.33 \%$ and Hodgkin s lymphomas for $4.16 \%$ each, while non- Hodgkin's lymphoma comprised the remaining $1.38 \%$. 
Similar observations were made by Jha B.C. et al., who studied 94 cases, of which tuberculosis was confirmed in $63.8 \%$ cases.

Age Distribution In Cervical Lymphadenopathy

Table 1. Age Distribution In Cervical Lymphadenopathy

\begin{tabular}{|l|l|l|}
\hline Age Group & No. of cases & percentage \\
\hline $11-20$ & 10 & 13.88 \\
\hline $21-30$ & 24 & 33.33 \\
\hline $31-40$ & 19 & 26.38 \\
\hline $41-50$ & 4 & 5.55 \\
\hline $51-60$ & 5 & 6.94 \\
\hline$>60$ & 4 & 5.55 \\
\hline Total & 72 & 100 \\
\hline
\end{tabular}

The commonest age group affected in the present study is 21-30 years accounting for $33.33 \%$ of cases, followed by 31-40years (26.38) and 11-20 years (13.88) The least affected age group was $>60$ years where only 4 cases were accounted. In the study by Jha B.C. et al., the commonest age group involved was 11-20 years, while study by Shafiullah et al. had $72 \%$ cases in the age group of 11-30.years, which is comparable with the present study. The study by Kim L.H. et al. comparable with present study, maximum number as it had of cases between 20-50 years of age.

Sex Distribution In Cervical Lymphadenopathy

Table 2 Sex Distribution In Cervical Lymphadenopathy

\begin{tabular}{|l|l|l|}
\hline \multicolumn{1}{|c|}{ SEX } & NUMBER OF CASES & PERCENTAGE \\
\hline MALE & 41 & 57 \\
\hline FEMALE & 31 & 43 \\
\hline TOTAL & 72 & 100 \\
\hline
\end{tabular}

Of the 72 cases, Most of the studies show female predilection. Few studies' like Purohit. S. D. et al. and Tripathy. S. N. et al. are comparable with this study.

41 cases were males 31 females. The sex ratio in the present study was $1.32: 1$ (M:F).

History Of Constitutional Symptom

Table 3 History Of Constitutional Symptom

\begin{tabular}{|l|l|l|}
\hline PRESENTING SYMPTOMS & NO OF CASES & PERCENTAGE \\
\hline Neck swelling & 52 & 72.2 \\
\hline Fever & 28 & 33.33 \\
\hline Change of voice & 3 & 4.1 \\
\hline Cough & 9 & 12.5 \\
\hline Malaise & 12 & 16.66 \\
\hline Difficulty is swallowing & 2 & 2.7 \\
\hline Loss of appetite & 7 & 9.7 \\
\hline Loss of weight & 15 & 20.8 \\
\hline
\end{tabular}

In the present study, only $50 \%$ of the cases with tuberculosis had constitutional symptoms. Similarly, only $33.33 \%$ of cases with malignant secondaries had symptoms. In comparison $75 \%$ \& $50 \%$ presented with symptoms in reactive/non specific lymph-adenitis and lymphomas respectively. Similar observations were made by JHA B. C et al and Jindal N. et al.

Site Distribution In Cervical Lymphadenopathy

In the present study, the Memorial Sloan-Kettering Hospital classification of neck lymph nodes from level 1 through level 7 was utilized. It was observed that in tuberculosis, the level 5 (posterior triangle group) was most commonly affected $(35.41 \%$ ) followed by level 2 (upper jugular group) at $27.08 \%$. About 11 cases $(22.91 \%$ ) had more than one site involved in the neck. In contrast, all cases $(100 \%)$ of lymphoma had more than one site involvement. In the Jha B.C. et al. series, upper deep jugular, group was the most commonly involved. In Baskota D.K. et al. study posterior triangle was the, commonest at $51 \%$ (comparable to the present study). In the Manolidis S.study anterior triangle (excluding submandibular) was the most commonly involved $(35.1 \%)$, similar percentage had multiple site involvement. Posterior triangle (including supra clavicular) came next with 9.1\% (again, comparable with present study). 


\section{Role Of FNAC In Cervical Lymphadenopathy}

The study by Jha B.C. et al. reported a sensitivity of $92.8 \%$ in diagnosing tubercular lymphadenitis. The study by Chao S.S., Loh K.S. et al. showed sensitivity of $88 \%$ and specificity of $96 \%$ for the same. Mondal A. et a1.83 reported $100 \%$ sensitivity in diagnosing tubercular and pyogenic lymphadenitis, and also Hodgkin's disease, 98\% for metastatic deposits; 97\% for chronic nonspecific lymphadenitis; $92 \%$ for Non-Hodgkin's lymphomas.

After studying 2216 cases, Prasad R.R. et at noted sensitivity and specificity of $84 \%$ and $95 \%$ respectively for tubercular lymphadenitis; $97 \%$ and $99 \%$..for metastatic deposits; $80 \%$ and $98 \%$ for Hodgkins disease; $81 \% \%$ and $96 \%$ for Non- Hodgkin's lymphomas. Comparable observations were made in the present study.

\section{Conclusion}

1) Tuberculosis is an important disease, one of the commonest disease affecting lymph nodes, it is curable with antituberculous drugs if administered as per the accepted regimen.

2) Clinical symptoms in cervical lymphadenopathy has limited significance and clinical behavior can be highly variable. Dependence on clinical evidence alone would lead to erroneous diagnosis in a considerable number of cases.

3) FNAC can be deemed as a frontline investigation' with further investigations on the basis of FNAC result. However, histopathological examination remains the most dependable diagnostic tool.

4) Most of the diseases are medically curable with limited role for surgery in non- neoplastic lesions.

5) Cervical lymphadenopathy is an important disease, commonly come across, and always calls for meticulous attention, analysis and treatment. Even though the problem is practically eradicated in Western countries it is still prevalent in India. Lymph node tuberculosis is a disease of great antiquity. It is commonest of extra-pulmonary tuberculosis and is probably the commonest cause of chronic lymphadenitis in children. Even after the advent of effective chemotherapy for tuberculosis, it still poses considerable problems in diagnosis and management. The disease even presents as painless lymphadenopathy of superficial lymph nodes of insidious onset. Head and neck cancers account for $2.8 \%$ of all newly discovered cancers. Metastatic carcinoma within cervical lymph nodes with an unknown primary tumour site accounts for 3\% to $5 \%$ of all head and neck cancers.

The control of such regional metastatic disease constitutes a significant part of the process of treating head and neck cancer. The presence of an enlarged node proven histologically positive for metastasis is an ominous finding and as a general rule decreases the 5 -year survival rate by at least $50 \%$. When nodal involvement becomes multiple extends low in the neck, no patient gets cured regardless of the treatment given the primary site of carcinoma is known, focused therapy to the primary site and cervical lymphadenopathy can be given. Without this knowledge clinicians are obligated to treat the entire pharyngeal axis and larynx to cover the possible origins of the metastatic carcinoma.

The occult primary treatment regimen results in a significant increase in morbidity to the patient. Proper understanding of the anatomy and detection of cervical metastatic disease is crucial to this process

\section{References}

[1]. Cheesman AD. Pharnyx, Larynx and Neck. Chapter-45 in: Bailey \& Loves Short Practice of Surgery, 25th Edition,

[2]. Farhi DC, Wells ST, Sigel RJ. Syphilitic lymphadenopathy - Histology arid human immunodeficiency virus status. Am. J. Clin Pathol.1999 Sept; 112(3): 330-4.

[3]. Schmid Geert, Schoenbein, John Ros Ir. Structure function relation in peripheral circulation. Chapter-6 in: Best $\&$ Taylor"s, Physiological basis of medical practices. $9^{\text {th }}$ Edition, West John B; New Delhi: 1996. 116

[4]. Jha B C,Dass A,. Cervical Tuberculous Lymphadenopathy: changing clinical patterns and concepts in management .Post - graduate Med. J 2001; Mar 7799050:185-7

[5]. Richard S Snell. The Head and Neck. Chapter-11. In: Clinical Anatomy for Medical Sciences. rd Edn. 1986. P. 743

[6]. Farhi DC, Wells SJ Siegel RJ. Syphilitic lymphadenopathy. Histology \& Human Immunodeficiency Virus Status. Am. J. Clin Pathol, 1999 Sept: 112(3): 330-4.

[7]. Shaha A, Webbere C, Marti J,Fine needle aspiration in the diagnosis of cervical lymphadenopathy .Am.j.Surg.1986 0ct ; 152[4]: 420-3.

[8]. Zeidman I, Buss JM, Experimental studies on the spread of cancer in the lymphatic system. 1. effectiveness of the lymph nodes as a barrier to the passage of embolic tumour cells. Cancer Res. 14: 403, 1994.

[9]. Osama G, Peter R, Charles E, Joseph J. Metastatic malignant disesase of unkown orgin . Am j Surg Pathol 145, 492-7.

[10]. Prasad RR, Narasimhan R, Shankaran V. FNAC in diagnosis of superficial lymphadenopathy of 2418 cases. Diag cytopathaol 1996 Dec;15(5);382-6. 